\title{
Small Intestinal B-Cell Non-Hodgkin Lymphoma
}

National Cancer Institute

\section{Source}

National Cancer Institute. Small Intestinal B-Cell Non-Hodgkin Lymphoma. NCI

Thesaurus. Code C96053.

A B-cell non-Hodgkin lymphoma that arises from the small intestine. Representative examples include diffuse large B-cell lymphoma, mucosa-associated lymphoid tissue lymphoma, alpha heavy chain disease/immunoproliferative small intestinal disease, Burkitt lymphoma, follicular lymphoma, and mantle cell lymphoma. 